\title{
Comparative Metabolomics of Transgenic Tobacco Plants (Nicotiana tabacum var. Xanthi) Reveals Differential Effects of Engineered Complete and Incomplete Flavonoid Pathways on the Metabolome
}

\author{
Corey D. Broeckling ${ }^{2}, \mathrm{Ke}^{-G a n g ~ \mathrm{Li}^{1} \text { and De-Yu Xie }}{ }^{1}$ \\ ${ }^{1}$ North Carolina State University, Department of Plant Biology, Raleigh, NC \\ ${ }^{2}$ Proteomics and Metabolomics Facility, Colorado State University, Fort Collins, CO
}

USA

\section{Introduction}

Anthocyanins and proanthocyanidins (PAs) are two groups of end products of the plant flavonoid pathway (Fig. 1). Biochemical and genetic evidences have demonstrated that they share the same upstream pathway beginning with phenylalanine through a series of enzymatic reaction to anthocyanidins. Anthocyanidins are either modified by glycosylation, methylation, or other reactions to form diverse anthocyanins (Springob et al. 2003) or catalyzed into flavan-3-ols by an anthocyanidin reductase (ANR) (Xie et al. 2003; Xie et al. 2006). In addition, leucoanthocyanidin reductase (LAR) has been enzymatically demonstrated to catalyze leucoanthocyanidins into catechin (Tanner et al. 2003). To date, whether or not this branch catalyzed by LAR exists in plants still remains open to genetic studies.

Production of Anthocyanin Pigment 1 (PAP1) encodes a R2R3-MYB transcription factor and is a master regulator of anthocyanin biosynthesis in Arabidopsis thaliana (Fig. 1) (Borevitz et al. 2000; Tohge et al. 2005; Xie et al. 2006). The constitutive expression of PAP1 in Arabidopsis and tobacco resulted in massive accumulation of targeted anthocyanins and numerous other phenylpropanoid compounds (Borevitz et al. 2000; Tohge et al. 2005). Our recent experiments revealed that the regulatory function of PAP1 on anthocyanin biosynthesis is closely associated with cellular specificity in tissues. In transgenic tobacco leaves of PAP1 and pap1-D Arabidopsis leaves, anthocyanins accumulated in epidermal cells and parenchymal cells of vascular bundles in veins (Shi and Xie 2010; Xie et al. 2006). It was interesting that the overexpression of PAP1 led to massive accumulation of anthocyanins in transgenic tobacco leaf trichomes (Xie et al. 2006) but not in leaf trichomes of pap1-D Arabidopsis thaliana (Shi and Xie 2010). In vitro separation of red cells from other cells has demonstrated that the regulatory function of PAP1 expression can be inherited by cell culture developed from mother plants (Zhou et al. 2008). Both no anthocyanin-producing white cells and anthocyanin-producing red cells were obtained from transgenic PAP1 
tobacco leaves. Transcript analysis showed that the level of the PAP1 overexpression in these two types of cells were similar, indicating that the regulatory function of the PAP1 expression was dependent upon cell types. (Zhou et al. 2008). The regulatory function of PAP1 is also highly controlled by environmental factors. Although the pap1-D Arabidopsis plants over express PAP1 leading to high anthocyanin pigmentation in most of tissues (Borevitz et al. 2000), when growth conditions were changed, anthocyanin levels and composition were dramatically altered in leaves of pap1-D plants (Rowan et al. 2009; Shi and Xie 2010; Tohge et al. 2005). In the same nutrition condition, high light conditions increased anthocyanin levels in leaves of pap1-D plants; under the same light condition, high nitrogen nutrition conditions increased anthocyanin levels in leaves of pap1-D plants; although in these conditions, the expression levels of PAP1 were similar (Shi and Xie 2010). These inconsistent relationships between the expression levels of PAP1 and anthocyanin levels were likely associated with other transcription factors involved in anthocyanin biosynthesis. PAP1 (MYB75), TT8 (transparent testa 8) (bHLH) / GL3 (glabra 3) (bHLH) and TTG1 (transparent testa glabra 1) (WD40) have been demonstrated to form a regulatory complex of MYB-bHLH-WD40 (MBW) controlling anthocyanin biosynthesis in Arabidopsis (Gonzalez et al. 2009; Ramsay and Glover 2005). We recently determined that the PAP1-TT8/GL3TTG1 complex independently regulated anthocyanin biosynthesis in pap1-D cells (Shi and Xie 2011). In addition, there are other MBW regulatory complexes controlling anthocyanin biosynthesis in Arabidopsis (Gonzalez et al. 2009). In different growth conditions, the regulatory function of $P A P 1$ is likely essentially dependent upon these regulatory complexes.

ANR is a NADPH/NADH-dependent flavonoid reductase converting anthocyanidins to flavan-3-ols (e.g. epicatechin) and PAs (Fig. 1) (Xie et al. 2003). ANR is encoded by a BANYULS gene that was first cloned from young seeds of Arabidopsis (Devic et al. 1999). Its homologs were cloned from different species including a model legume plant Medicago truncatula (Xie et al. 2003). The constitutively ectopic expression of ANR in tobacco showed the loss of anthocyanins in flowers, in which ANR competitively catalyzed anthocyanidins into flavan-3-ols (e.g. epicatechin) and PAs (Xie et al. 2003). The transgenic vegetative tissues including leaf and stem tissues also expressed ANR, but failed to form epicatechin and PAs due to the absence of anthocyanidins (Xie et al. 2003; Xie et al. 2006). To establish a complete pathway of PAs in ANR transgenic leaves and stems, PAP1 were ectopically expressed in them. The co-expression of ANR and PAP1 produced flavan-3-ols and PAs in leaves and stems of transgenic tobacco plants (Xie et al. 2006). This result demonstrated that the expression of ANR alone resulted in production of ANR protein in transgenic leaves and stems and these tissues contained an incomplete PA pathway. This result also demonstrated that the overexpression of PAP1 provided substrates for ANR; thus formed an effective platform for metabolic engineering of flavan-3-ols and PAs.

Whether the ectopic expression of PAP1, ANR, and PAP1::ANR, which form different pathways in transgenic tobacco vegetative tissues, can impact other metabolisms beyond the flavonoid pathway remains unknown. In this study, metabolic profiles of wild-type (WT) and transgenic tobacco plants expressing PAP1, ANR, and PAP1::ANR were examined by GC-MS analysis, showing that these three transgenic events differentially altered accumulation patterns of both targeted and non-targeted metabolites beyond anthocyanins and PAs in both transgenic leaves and stems 


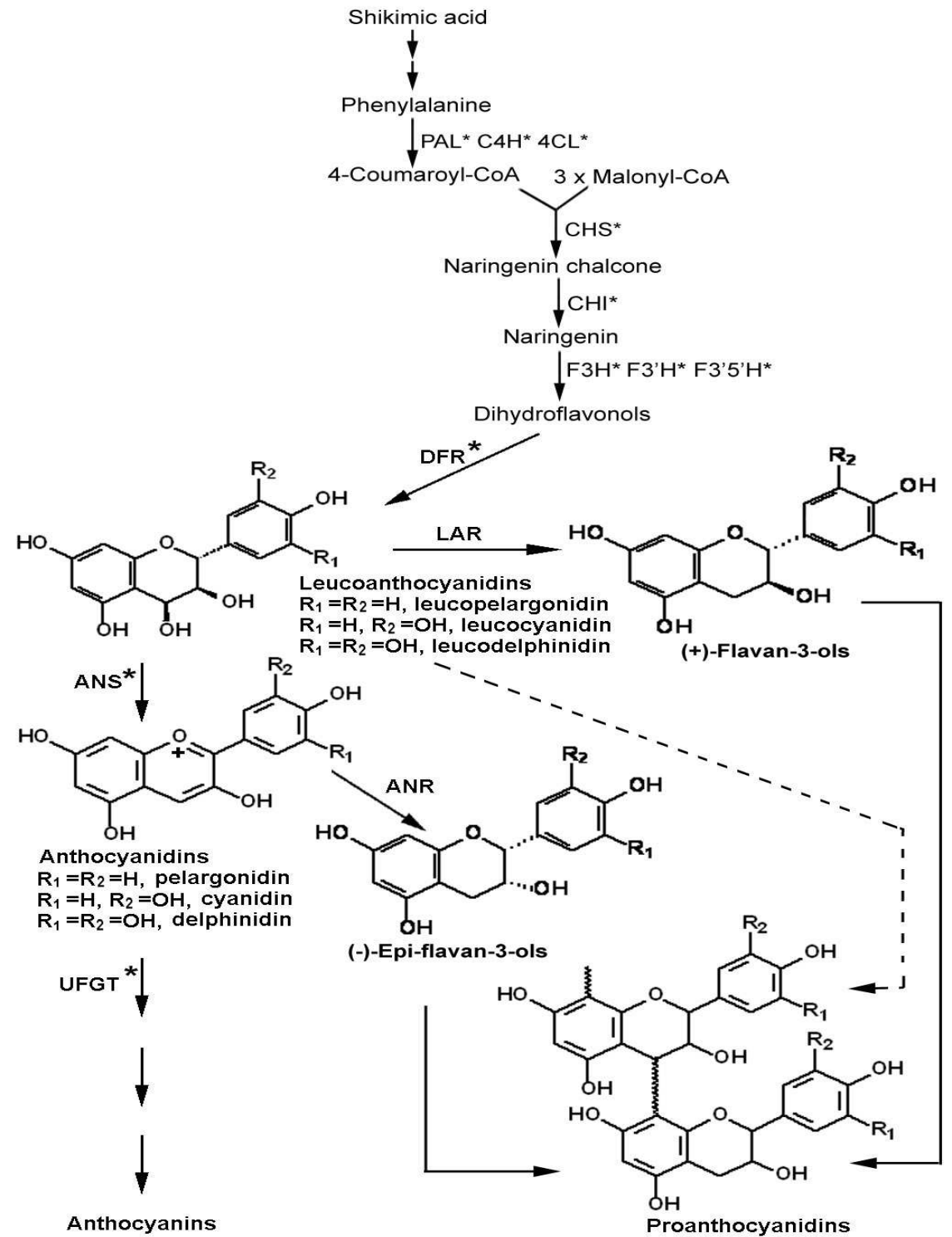

Fig. 1. Biosynthetic pathways of anthocyanins and proanthocyanidins starting with shikimic acid. Enzymes include: PAL, L-phenylalanine ammonia-lyase; $\mathrm{C} 4 \mathrm{H}$, cinnamate-4hydroxylase; 4CL, 4-coumarate: $\mathrm{CoA}$-ligase; $\mathrm{CHS}$, chalcone synthase; $\mathrm{CHI}$, chalcone isomerase; F3H, flavanone-3-hydroxylase; F3'H, flavonoid 3'-hydroxylase; F3'5' H, flavonoid 3', 5'-hydroxylase; DFR, dihydroflavonol reductase; ANS, anthocyanidin synthase; ANR, anthocyanidin reductase; UFGT: uridine diphosphate glucose-flavonoid 3-Oglucosyltransferase. Two arrows between shikimic acid and phenylalanine means there are multiple steps. Asterisks $\left(^{*}\right)$ indicate steps known to be up-regulated by PAP1 expression. 


\section{Materials and methods}

\subsection{Plant growth}

Plant materials used for this experiment included PAP1, ANR and PAP1:: ANR (F1 progeny) transgenic plants as well as wild-type (WT) plants. WT plants was the control line \#3, which has been used as control plants to characterize PAP1 gene function in metabolic engineering of PAs (Xie et al. 2006). PAP1 transgenic plants containing an engineered pathway of anthocyanins were a homozygotic one from the line \#292 (Xie et al. 2006). The ANR transgenic plants were derived from the line \# B-21, which has only one copy of the MtANR (Medicago truncatula ANR) transgene and produces PAs in flowers, (Xie et al. 2003), but contains an incomplete pathway of PAs in leaves and stems. The PAP1::ANR transgenic plants were derived from the line \# P-B-13 (PAP1 x ANR) that was obtained by crossing the line \# B-21 with the line \#292 of PAP1 transgenic plants. This line produced a relatively high level of PAs in leaves and stems resulting from an engineered PA pathway (Xie et al. 2006).
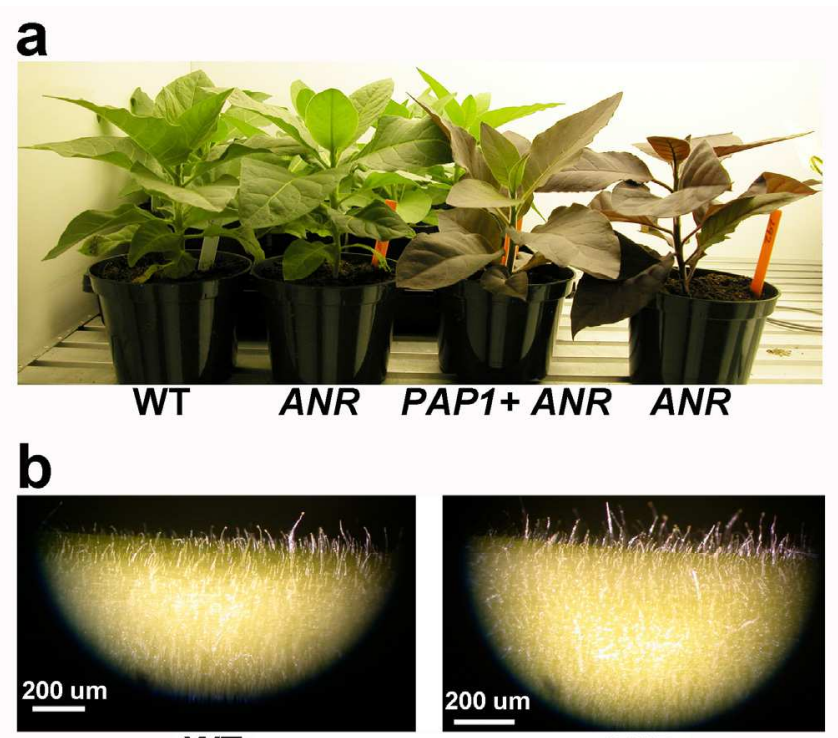

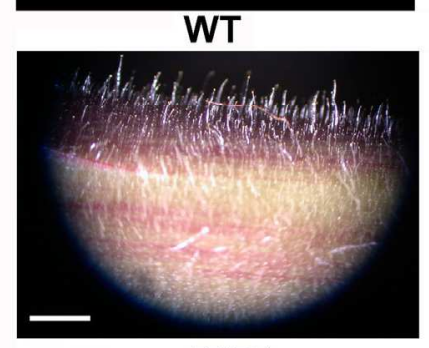

PAP1

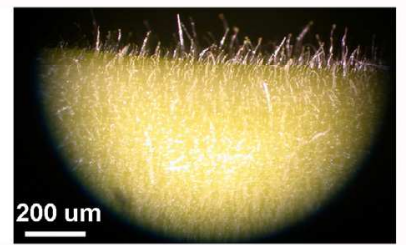

ANR

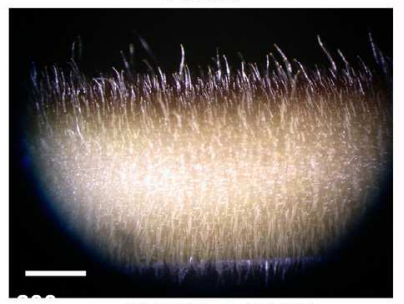

PAP1 +ANR

Fig. 2. Phenotypes of plants and stem trichomes. a phenotypes of wild-type (WT) and transgenic tobacco plants; $\mathrm{b}$ trichomes from stems; WT: wild type; $A N R, P A P 1$, and PAP1 + ANR: ANR, PAP1, and PAP1::ANR transgenic plants. 
Ten individual plants from each of four genetic background lines were vegetatively propagated by cutting. Finally, 37 plants (10 for wild-type and PAP1 and PAP1::ANR transgenic plants, respectively, and 7 for $A N R$ transgenic plants) were grown in a growth chamber for metabolic profiling. To minimize effects of physical factors (e.g. temperature, lighting, and humidity) on in vivo biochemical variability, plants were grown in pots with a size of three inches in radius and eight inches in height in a growth chamber at a constant temperature of $25^{\circ} \mathrm{C}, 70 \%$ humidity, $16: 8 \mathrm{hr}$ light: dark cycle, and under fluorescent epi-illumination. The possible effects of shading on plant growth were reduced by rotating the pots. The growth of all plants was morphologically similar (Fig. 2 a).

Sixty-two day old plants, which were approximately $30 \mathrm{~cm}$ in height with 10-13 leaves, were used for metabolome analysis. Plant tissues were harvested directly into liquid nitrogen. Plant tissues collected included old leaves, young leaves, and stems. Old leaves were defined as those that were fully expanded, $15-20 \mathrm{~cm}$ in length, from each individual plant, while young leaves were defined as those with $5-10 \mathrm{~cm}$ in length, not yet fully expanded. Old leaves, young leaves or stems from a single plant was pooled to create one sample, thus 111 samples in total were obtained from 37 plants. The frozen samples were stored at $-80^{\circ} \mathrm{C}$, freeze-dried at $-80^{\circ} \mathrm{C}$, and then ground into a fine powder, which was stored at $-80^{\circ} \mathrm{C}$ until use.

\subsection{Tissue extraction and GC-MS analyses}

A polar and a lipophilic extraction for each sample were profiled in this experiment. Metabolites were extracted from lyophilized plant material as described previously (Broeckling et al. 2005). Briefly, freeze-dried homogenized tissue $(6.0+/-0.05 \mathrm{mg})$ was weighed into a vial $(4.0 \mathrm{ml})$ for extraction of metabolites by adding $\mathrm{CHCl}_{3}(1.5 \mathrm{ml})$ that contained an internal standard (heptadecanoic acid methyl ester). Vials were thoroughly vortexed and incubated at $50^{\circ} \mathrm{C}$ for $45 \mathrm{~min}$, followed by addition of HPLC grade $\mathrm{H}_{2} \mathrm{O}(1.5$ $\mathrm{ml}$ ) containing a second internal standard (ribitol). The biphasic system was thoroughly vortexed and then incubated for an additional $45 \mathrm{~min}$ at $50^{\circ} \mathrm{C}$. The sample was then centrifuged at $3000 \times g$ for $30 \mathrm{~min}$ at $4^{\circ} \mathrm{C}$. One $\mathrm{ml}$ of each phase was collected and the solvent was then evaporated either using speed vacuum (aqueous phase) or dried under liquid nitrogen stream $\left(\mathrm{CHCl}_{3}\right.$ phase). The polar phase extraction was methoximated in $120 \mu \mathrm{l}$ of $15 \mu \mathrm{g} / \mu \mathrm{l}$ methoxyamine $\mathrm{HCl}$ in pyridine for $120 \mathrm{~min}$ at $50^{\circ} \mathrm{C}$ and then trimethylsilyalted by adding $120 \mu \mathrm{l}$ of MSTFA $+1 \%$ TMCS (Pierce Biotechnology, Rockford, IL, USA) and incubating for $60 \mathrm{~min}$ at $50^{\circ} \mathrm{C}$. The non-polar phase extraction was derivitized in $100 \mu \mathrm{l}$ of $50 \%$ MSTFA $+1 \%$ TMCS in pyridine at $50^{\circ} \mathrm{C}$ for $60 \mathrm{~min}$. One $\mu \mathrm{l}$ of each was injected onto an Agilent 6890 GC coupled to a 5973 MS. The polar sample was split at 15:1 and the non-polar sample split $1: 1$. The oven program was $80^{\circ} \mathrm{C}(2 \mathrm{~min})$ and ramped at $5^{\circ} \mathrm{C} / \mathrm{min}$ to $315^{\circ} \mathrm{C}(12$ $\mathrm{min}$ ). Separation was performed on a 60m DB-5MS (J\&W Scientific $-0.25 \mathrm{~mm}$ ID and 0.25 $\mu \mathrm{M}$ film thickness) at a flow rate of $1.0 \mathrm{ml} / \mathrm{min}$. Metabolites were identified by comparison to a library of electron impact mass spectra and GC retention time as described previously (Broeckling et al. 2005). Identification was performed by using AMDIS deconvolution and identification software (NIST). The same GC separation parameters were used for obtaining positive and negative-ion chemical ionization spectra for tentative identification of the 
cembratienols using methane as the ionization gas on an Agilent 5973N MS. Quantification was performed as described previously (Broeckling et al. 2005).

\subsection{Data processing, statistical analysis and heatmap}

Peak detection and deconvolution were performed with AMDIS (Halket et al. 1999) for 23 samples of each treatment. Resultant peak lists were imported and compiled in METIDEA (Broeckling et al. 2006) and then used to extract quantitative peak area values for polar and non-polar metabolites. Redundant peaks were removed from the dataset, peak area values were scaled to mean zero and standard deviation 1.0. The resulting data matrix was statistically analyzed with discriminant function analysis, ANOVA, and principal component analysis in JMP (SAS institute, Cary, North Carolina). One-Way ANOVA was performed to extract significant different levels of metabolites ( $p$-values < 0.05), then followed by Tukey's HSD pos-hoc analysis to compare all pair-wise mean difference ( $p$-value $<0.05$ ). To visualize metabolite accumulation patterns and the effect of transgenes on metabolite levels in tissues, heatmaps were established from excel using vision functions in macro.

\subsection{Histological analysis of anthocyanin accumulation}

Both young leaves and stems were dissected to $10-20 \mu \mathrm{m}$ in thickness by hands and cellular localization of anthocyanin accumulation was examined with light microscope as described previously (Xie et al. 2006).

\section{Results}

\subsection{Plant growth and cellular localization patterns of anthocyanin accumulation}

The growth of all plants was morphologically similar in the growth chamber except for color difference (Fig. 2 a). Red pigmentation patterns were examined with a microscope. Trichomes, epidermis, and hypodermis of PAP1 transgenic stems highly accumulated anthocyanins (Figs. 2 b and 3). Parenchyma cells around vascular bundle and particularly near phloem were clearly red or pink resulting from high accumulation of anthocyanins (Fig. 3 b, c and e). In addition, parenchymal cells around xylem were clearly red or pink due to the high accumulation of anthocyanins (Fig. 3 b, c and e). Cells in the pith and cortex were not observed to produce anthocyanins (Fig. 3 b, c and e). In contrast, all cells in WT stems did not produce anthocyanins in the same growth conditions (Fig. 2 a-b and Fig. $3 \mathrm{a}, \mathrm{d}$ and f). In addition, ANR transgenic plants did not produce anthocyanins either (Fig. 2 a and b). As reported previously for greenhouse-grown plants (Xie et al. 2006), $P A P 1:: A N R$ transgenic plants showed obviously reduced levels of anthocyanins in plants (Fig. $2 \mathrm{a}$ and $b$ ).

We previously reported the features of anthocyanin accumulation in PAP1 transgenic leaves of greenhouse-grown plants. Anthocyanins were mainly localized in trichomes, epidermal cells, hypodermal cells, and parenchyma cells in veins (Xie et al. 2006). In this experiment, the accumulation patterns of anthocyanin in leaves were the same as ones in leaves of greenhouse-grown plants (Xie et al. 2006). 


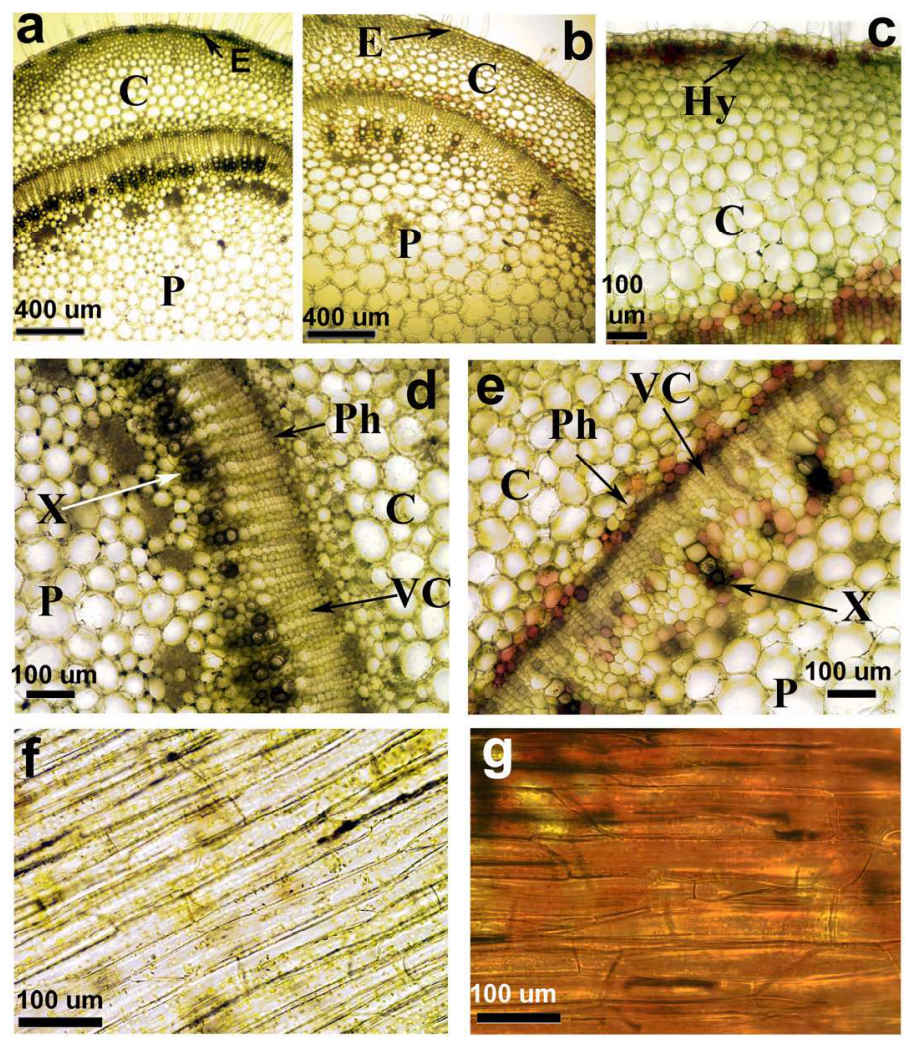

Fig. 3. Microscopic images show cellular specificity of anthocyanin accumulation in stems of PAP1 transgenic plants. $a-b$ cross sections of young stems of wild-type (a) and PAP1 transgenic (b) plants; $c$ a magnified image showing anthocyanin accumulation in hypodermal cells of PAP1 transgenic stems; d a magnified image showing vascular bundle of WT stems; e a magnified image showing anthocyanin accumulation patterns in or around vascular bundles of PAP1 transgenic stems; f epidermal cells of WT stems; g epidermal cells of PAP1 transgenic stems. Abbreviations: C, cortex; E, epidermis; Hy: hypodermis; P: pith; Ph: phloem; VC: vascular cambium; X: xylem.

\subsection{Metabolites identified and their profile properties in wild-type tobacco}

Young leaves, old leaves, and stem tissues of WT tobacco plants were used to analyze metabolites and examine their accumulation patterns. In total, eighty-seven metabolites including both water (polar molecular compounds) and chloroform soluble compounds (low or non-polar molecular compounds) were characterized based on their mass spectra profile identity and chromatographic retention times in comparison to authentic standards. ANOVA significance tests indicated that seventy-one metabolites were featured with tissuerelated accumulation patterns, which was shown in a heatmap (Fig. 4). Fourteen metabolites did not show significant differences among the three tissues examined. In addition, two metabolites, ribose and trihydroxybutyrate showed an interesting pattern in the three 
tissues. ANOVA analysis showed that the levels of the two compounds were not obviously different between young leaves and stems as well as between old leaves and stems but significantly higher in young leaves than in old ones.

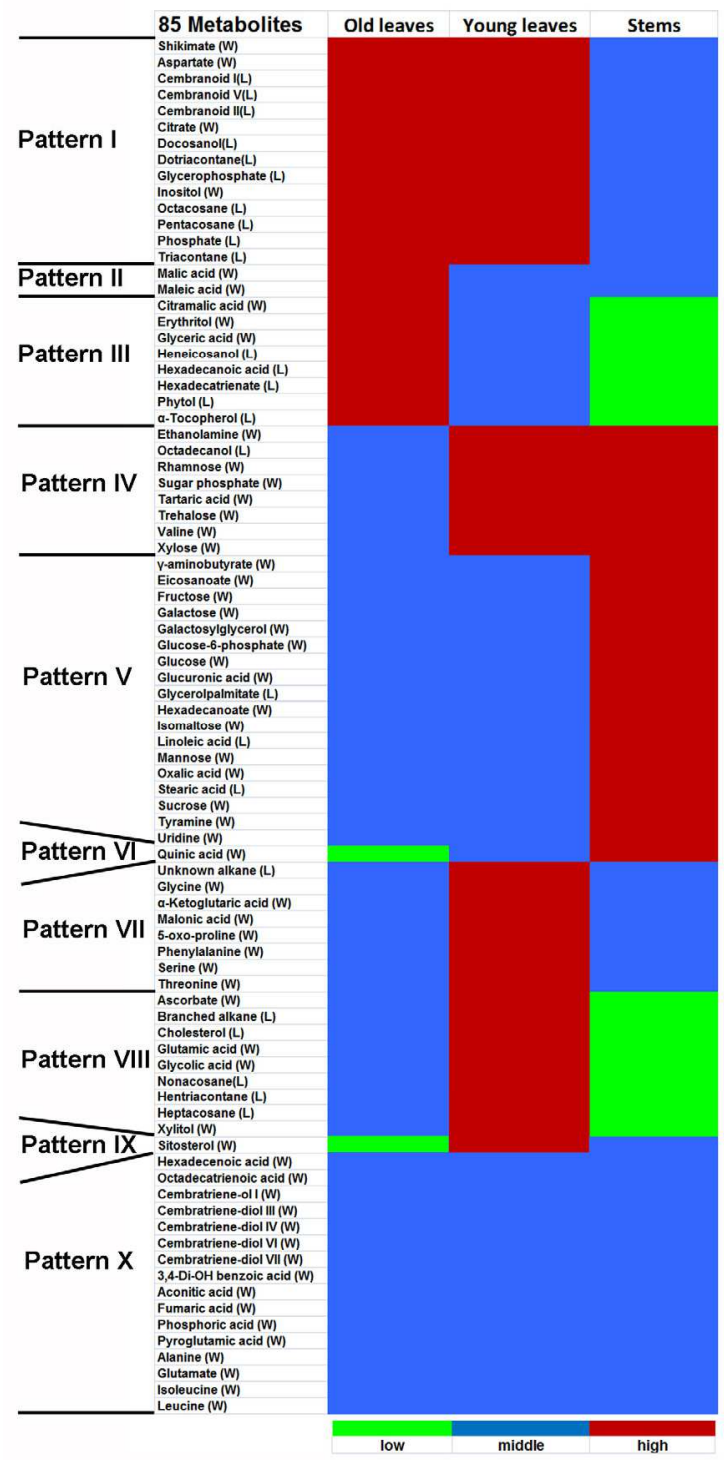

Fig. 4. A heatmap shows differential accumulation patterns of 85 metabolites in stems, young leaves, and old leaves. In each pattern, red color means the highest levels of metabolites in the tissue $(\mathrm{P}<0.05)$, and blue color means significantly $(\mathrm{P}<0.05)$ higher levels of metabolites in the tissue than in the other tissue indicated by green colors. (L): lipophilic metabolites extracted in chloroform phase; $(\mathrm{W})$ : water soluble metabolites in extracted in water phase. 


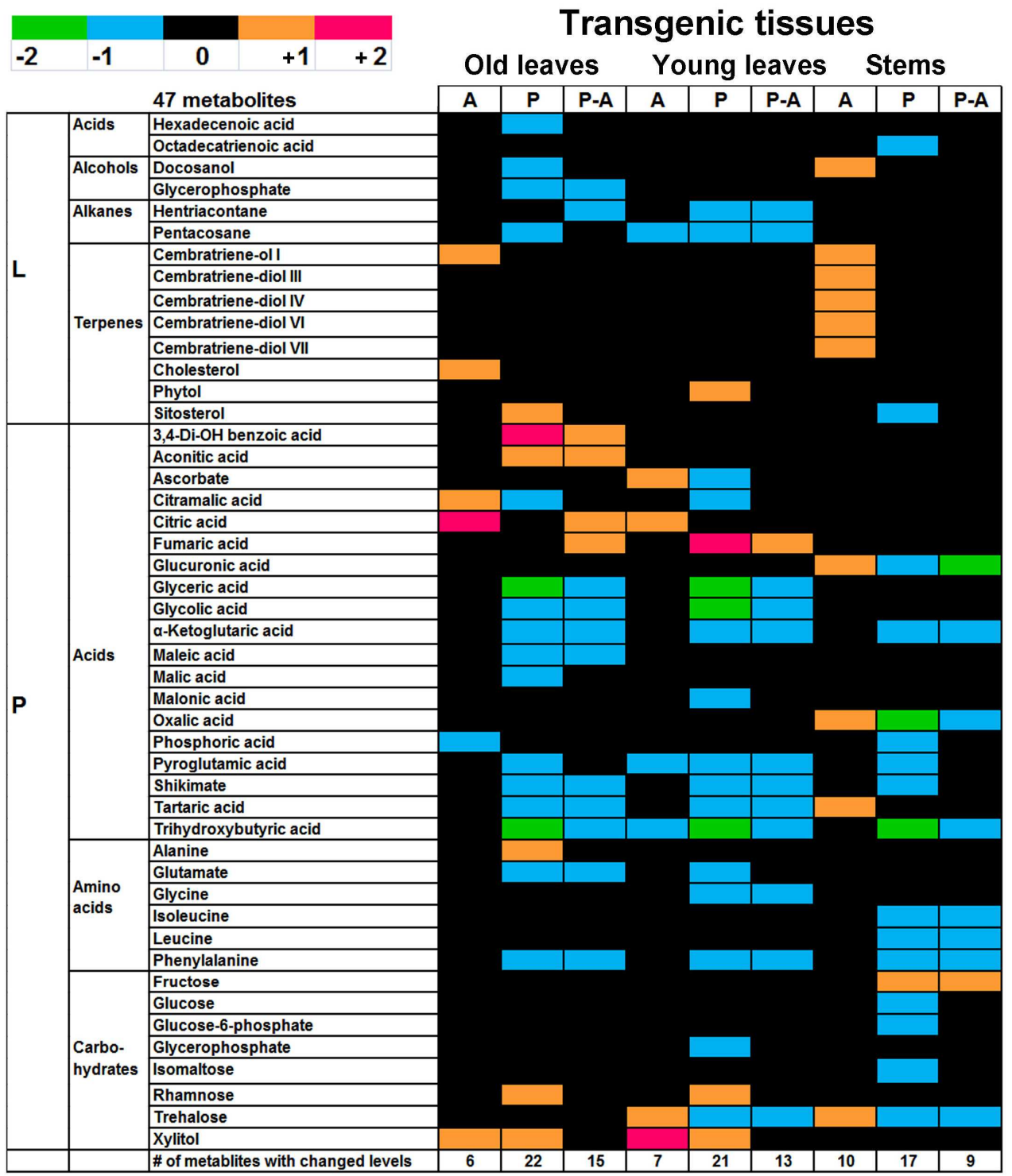

Fig. 5 A heatmap shows significant increase or decrease of levels for metabolite in PAP1, $A N R$, and PAP1::ANR transgenic stems and young and old leaves. Black color (0) indicates no differences in levels of metabolites between transgenic tissues and wild-type tissues. Red $(+2)$ color indicates highly significant $(\mathrm{P}<0.05)$ increase. Bright brown color $(+1)$ indicates significant $(\mathrm{P}<0.05)$ increase. Blue color $(-1)$ indicates significant $(\mathrm{P}<0.01)$ decrease. Green color $(-2)$ indicates highly significant $(\mathrm{P}<0.05)$ decrease. A: ANR; P: PAP1; P-A: PAP1:: ANR; $\mathrm{L}$ : lipophilic metabolites extracted in chloroform phase; $\mathrm{W}$ : water soluble metabolites in extracted in water phase. 


\subsection{Metabolites with significantly altered levels in PAP1 transgenic plants}

PAP1 is a master transcriptional regulator of the anthocyanin pathway (Fig. 1) (Xie et al. 2006). PAP1 transgenic plants highly produced anthocyanins in the growth chamber (Fig. 2 a). GC-MS analysis of the eighty-seven metabolites showed that the levels of thirty-nine were altered, which included nine metabolites with increased levels, twenty-nine metabolites with reduced levels, and one metabolite, sitosterol, the level of which was increased in old leaf but decreased in stem (Fig. 5).

Phenylalanine, malonic acid and shikimic acid, which are three early pathway precursors of the anthocyanin biosynthesis (Fig. 1), were dramatically reduced (Fig. 5). The decreases of phenylalanine and shikimic acid were apparent in all three analyzed tissues, while malonic acid was significantly reduced only in young leaves (Fig. 5).

Thirty-six metabolites with altered levels were not immediately metabolically related to plant phenylpropanoids (Fig. 5). These metabolites included two clusters consisting of seven non-polar and twenty-nine polar compounds either in old leaves, young leaves, or stems. The seven non-polar metabolites, which were composed of acids, alkanes, alcohols, and terpenes, included 4 in old leaf, 3 in young leaf, and 2 in stem. For example, the level of nonpolar compound docosanol was increased only in old leaf, while the abundance of nonpolar compound sitosterol was reduced in stem but increased in old leaf. The twenty-nine polar metabolites, which were composed of acids, amino acids, and carbohydrates, included 18 in old leaf, 18 in young leaf, and 15 in stem. Examples of decreased accumulation included a-ketoglutaric acid, citramalic acid, glyceric acid, glycine, maleic acid, and trihydroxybutyric acid in a tissue specific fashion.

\subsection{Metabolites with altered levels in ANR transgenic plants}

Our previous work discovered that ANR (anthocyanidin reductase) was a pathway enzyme of the PA biosynthesis (Xie et al. 2003) . Analyses of variance followed by Tukey's HSD posthoc comparisons were conducted to assess the alteration extent of metabolites in ANR transgenic plants. The levels of nineteen identified metabolites including eleven polar and eight non-polar metabolites were altered in tissue-dependent accumulation patterns (Fig. 5). The eleven polar metabolites included 4 in old leaf, 6 in young leaf, and 4 in stem, the levels of eight of which were increased in either leaves or stems, while, the levels of three of which were decreased in ANR transgenic leaves. The eight non-polar metabolites included seven with increased levels but one, pentacosane (a lipophilic odd-chained alkane) with a decreased level (Fig. 5). Among the seven metabolites with increased levels, cembratriene-ol I, cembratriene-diol III, cembratriene-diol IV, cembratriene-diol VI, and cembratriene-diol VII are defensive compounds against aphids (Wang et al. 2001).

\subsection{Metabolites with altered levels in PAP1: ANR transgenic plants}

$P A P 1:: A N R$ transgenic plants were the $\mathrm{F} 1$ hybrid progeny of ANR and PAP1 transgenic plants. These plants formed both anthocyanins and PAs (Xie et al. 2006). The levels of twenty-four metabolites including three non-polar and twenty-one polar metabolites were altered (Fig. 5). Among them, twenty-three were included in those metabolites with altered levels in PAP1 transgenic plants as described above (Figs. 5 and 6). Phenylalanine and shikimic acid are two early precursors of the anthocyanin and PA pathways (Fig. 1). As observed in PAP1 transgenic plants described above, the accumulation level of 
phenylalanine was significantly reduced in all analyzed tissues. The accumulation level of shikimic acid was also dramatically reduced in leaf tissues. Twenty-two metabolites with altered levels were not directly related to plant flavonoid biosynthesis, twenty-one of which were included in those impacted byPAP1alone transgenic plants (Fig. 5). Therefore, these results supported the metabolite profile alteration in PAP1 alone transgenic plants.

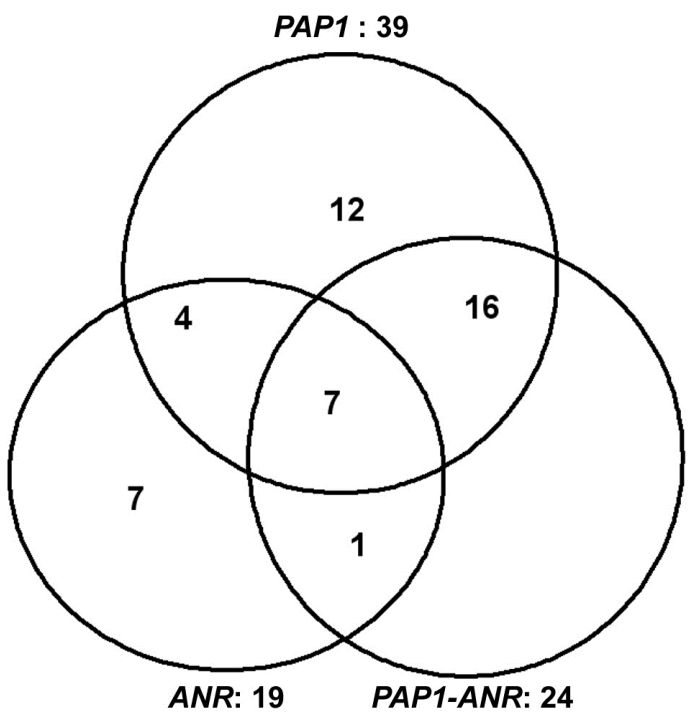

Fig. 6. A Venn diagram shows separate and overlaid properties of metabolites with altered levels in the PAP1, ANR, and PAP1::ANR (PAP1-ANR) transgenic plants. In total, the levels of forty-seven out of eighty-seven metabolites analyzed were impacted in the three transgenic events.

\subsection{Principal component analysis}

Principal component analysis (PCA) was performed with the eighty seven identified metabolites to examine the ordination relationships for all three tissues between WT plants and transgenic plants. The first principal component accounted for nearly 25\%, 23\% and $20 \%$ of total variation in old leaves, young leaves and stems respectively. The second principal component was nearly $12 \%$ of total variation in old leaves, young leaves, and stems. From PCA results for old and young leaves, it was obvious that PAP1 transgenic plants and WT type plants were clearly separated each other in the first principal component (Fig. $7 \mathrm{a}$ and b). ANR transgenic plants were scattered in the same cluster with WT plants. PAP1::ANR transgenic plants were between PAP1 transgenic and ANR transgenic or WT plants (Fig. $7 \mathrm{a}$ and b). It was interesting that in stems the ordination relationship patterns of four groups of plants were different from those observed in old and young leaves (Fig. 7 c). PAP1 and ANR transgenic stems were of the most separation in the first principal component. WT and PAP1 transgenic stems were also clearly different each other in the first principal component. As expected, PAP1 and PAP1::ANR transgenic stems were relatively close (Fig. $7 \mathrm{c}$ ). These PCA results showed that the impacts of PAP1 and ANR on metabolite profiles in transgenic plants were different. 
a

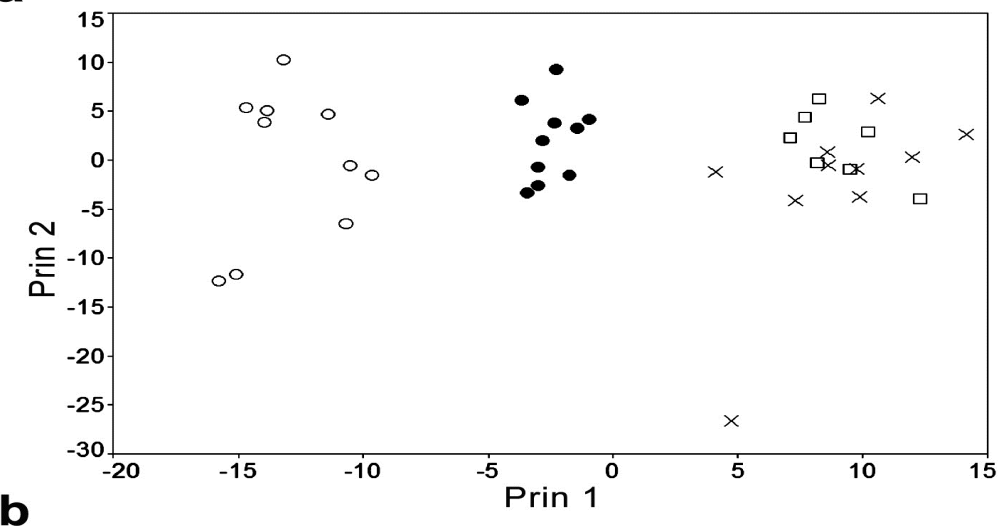

b
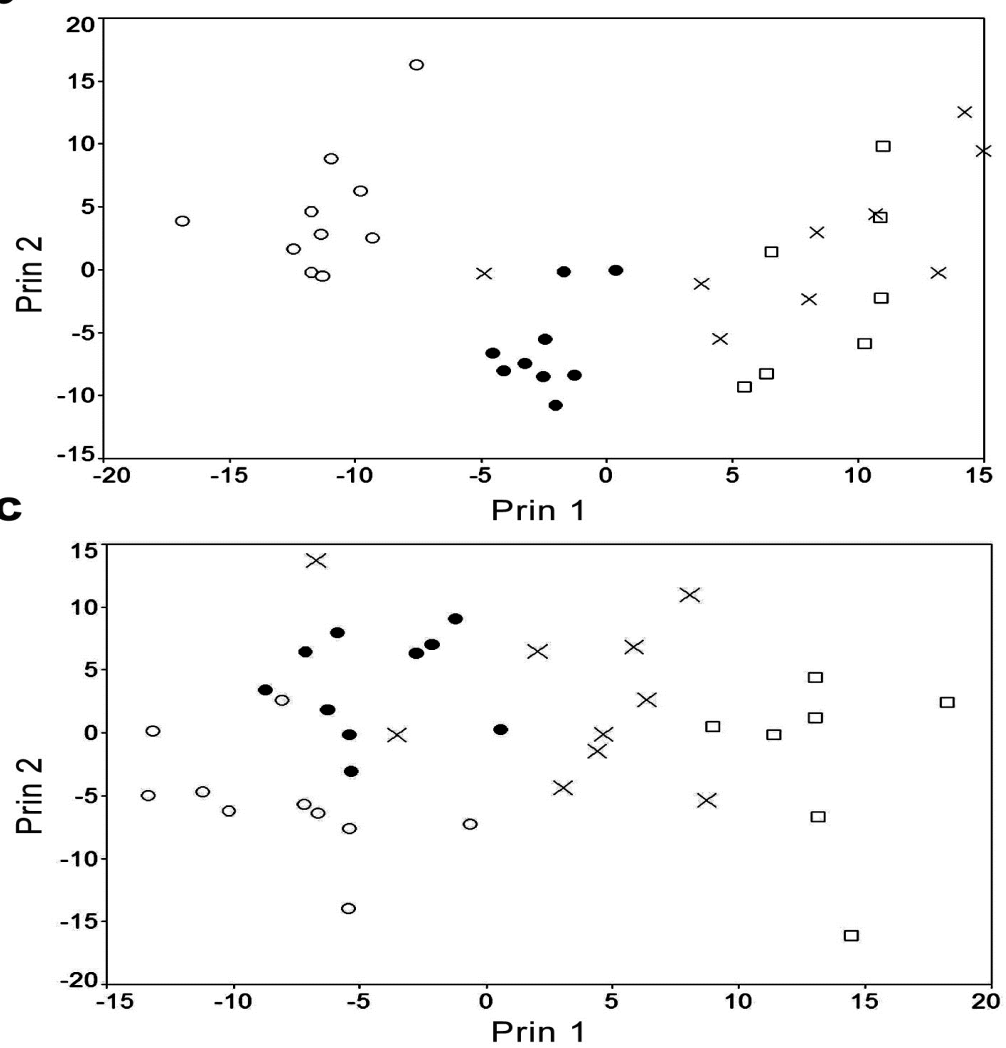

Fig. 7. Principal component analysis of wild-type plants ("x"), ANR (empty squares), PAP1 (empty circles), or PAP1::ANR (solid circles) for (a) old leaf, (b) young leaf, and (c) stem tissue. Principal components 1 and 2 account for (a) 24.5 and $12.1 \%$ of the total variation in old leaf, (b) 23.1 and $12.1 \%$ of the total variation in young leaf, and (c) 20.2 and $11.8 \%$ of the variation in stem tissue samples. 


\section{Discussion}

\subsection{Impacts of PAP1 transgene on metabolic profiles}

Our recent report showed that genome-wide transcriptional programs in red pap1-D Arabidopsis cells cultured in vitro were dramatically different from cultured wild-type cells (Shi and Xie 2011). On the one hand, massive numbers of genes involved in multiple metabolic networks were highly expressed in red cells. On the other hand, the expression levels of large number of genes involved in multiple metabolic networks were also reduced in red cells. We hypothesized that the dramatic alterations of genome-wide gene expression must have led to massive alterations of metabolic profiles beyond anthocyanin biosynthesis in red cells and specifically programmed red cells established by the overexpression of PAP1 must have led to transcriptional and metabolic reprogramming (Shi and Xie 2011).

In this study, GC-MS analysis revealed that levels of thirty-nine metabolites excluding flavonoids were altered in PAP1 transgenic plants (Figs 5 and 6). These metabolites are composed of seven non-polar molecules and thirty-two molecules including sugars, amino acids, and organic acids (Fig. 5). Based on their roles in anthocyanin biosynthesis, these metabolites can be characterized as targeted and non-targeted metabolites. Targeted metabolites are precursors in the anthocyanin pathway (Fig. 1). Phenylalanine, shikimic acid, and malonic acid are three early precursors of the anthocyanin biosynthesis. Therefore, the high production of anthocyanins must result from an enhanced metabolic flux from shikimic acid to anthocyanins (Fig. 1) and thus lead to the high consumption of shikimic acid, phenylalanine, and malonic acid. As expected, the levels of the three metabolites were reduced in PAP1 transgenic plants. This result metabolically supported the previous reports about the effect of PAP1 on gene expression profiles at the transcriptional level involved in anthocyanin biosynthesis. The transcriptional levels of the PAL1 and several other anthocyanin pathway genes were increased 1.2-6.7 folds by the overexpression of the PAP1 gene (Borevitz et al. 2000; Lillo et al. 2008; Tohge et al. 2005). In addition, this result supported our recent report that most of the pathway genes involved in the anthocyanin pathway in red cells over expressing PAP1 were dramatically increased leading to alterations of multiple metabolic networks (Shi and Xie 2011). Therefore, the alterations of metabolic profiles of the three molecules resulted from the ectopic expression of PAP1 transgene.

Most of metabolites with altered levels in PAP1 transgenic tissues were not directly related to the biosynthesis of anthocyanins. They are non-targeted metabolites. These metabolites included acids, amino acids, and carbohydrates (Fig. 5). Two studies showed that the transcriptional levels of several genes involved in sugar transport, glutathione metabolism, calcium binding, and carbohydrate metabolism were up-regulated by PAP1 in Arabidopsis (Borevitz et al. 2000; Tohge et al. 2005). However, no changes in the levels of amino acids, sugars, or anions profiled by capillary electrophoresis or liquid chromatography were observed (Tohge et al. 2005). This result was not in agreement with our current observation. We observed dramatic reduction of levels of several amino acids and carbohydrates in response to PAP1 expression in transgenic tobacco plants (Fig. 5). The discrepancy likely resulted from different experimental designs. We separated young leaves from old leaves in our experiments, while Tohge and co-workers (2005) did not in their study. More importantly, we recently isolated red cells from pap1-D Arabidopsis. Those red cells showed 
dramatically different transcriptional profiles from wild-type cells (Shi and Xie 2011) and these global gene expression alterations were not observed in Tohge's experiments either. Particularly, in red cells, expression levels of a large number of genes involved in glycolysis, amino metabolisms, photosynthesis and other metabolic pathways were significantly decreased (Shi and Xie 2011). In addition, the discrepancy might result from the speciesspecific responses to the over-expressed PAP1 protein. The results of ectopic expression of the A. thaliana PAP1 gene in tobacco plants might differ from the consequences of its overexpression in its native plant species. The evidence was that we recently observed dramatically differential response of anthocyanin biosynthesis to chemical factors in tissue cultures of PAP1 transgenic Arabidopsis and tobacco (Shi and Xie 2011; Zhou et al. 2008). Therefore, the alterations of these non-targeted metabolite profiles most likely resulted from the ectopic expression of PAP1 transgene.

\subsection{Impacts of ANR transgene on metabolic profiles}

It was very interesting that our data showed the altered levels of nineteen metabolites in leaf and stem tissues of the ANR transgenic plants. Obviously, the accumulation of five cembranoids was enhanced in the stem of the ANR alone transgenic plants (Fig. 5). Cembranoids are diterpenoids derived from the isoprenoid pathway and involved in defense against aphids (Wang et al. 2001). In Nicotiana spp., relatively high levels of cembranoids were found to localize to secretory glandular trichomes (Wang et al. 2004; Wang et al. 2001). In addition, oxalic acid accumulated at higher levels in ANR plants in a pattern similar to that of the cembranoids. Oxalate, which accumulates in glandular trichomes and idioblast cells of Nicotiana species (Choi et al. 2001; Sarret et al. 2006; VolkA and Franceschi 2000), is the calcium salt of oxalic acid and has important defensive activities against chewing insects (Korth et al. 2006). We microscopically examined trichomes, but did not observe changes of their morphology and density on the surfaces of stems and leaves between transgenic and WT tobacco plants (Fig. $2 \mathrm{~b}$ ), suggesting that their level increase most likely resulted from the ANR transgene expression.

The mechanism behind the metabolic alteration in ANR transgenic plants is unclear. Little has been reported regarding the mechanism through which ectopic expression of a single biosynthetic reductase can dramatically alter cellular metabolome. Here, we would provide certain perspective discussion to potentially interpret this result observed from our experiments. A few studies revealed the effects of marker genes on in vivo cellular biochemical processes. Constitutive expression of marker genes such as NPTII and GUS genes resulted in changes of certain transcriptome profiles in transgenic A. thaliana, although there was no impact on the development and growth of plants (Ouakfaoui and Miki 2005). The constitutive presence of Bt protein was observed to unexpectedly increase the production of lignin in insect-resistant transgenic corn plants (Saxena and Stotzky 2001). However, the mechanism of such observed effects caused by the presence of introduced foreign proteins has not been investigated in detail thus remains unknown. It is interesting that a report demonstrated that an overexpression of a rice dihydroflavonol reductase (DFR) led to enhanced levels of NAD $(\mathrm{H})$ and NADP $(\mathrm{H})$ in rice cell suspension and seedlings, which increased the tolerance of transgenic rice to bacterial pathogens and reactive oxygen species (ROS)-induced cell death (Hayashi et al. 2005). DFR is a NADPH/NADH dependent 
reductase essential for both anthocyanin and PA biosynthesis (Fig. 1) (Xie et al. 2004a). Although Hayashi et al (2005) did not report whether or not their transgenic rice seedlings and cells had in vivo substrates for DFR enzyme, nor did they measure changes in unrelated metabolic pathways, the constitutive presence of DFR protein increased the levels of the two coenzymes by enhancing the enzymatic activities of NAD synthetase, NAD kinase and ATPNMN adenylyltransferase (Hayashi et al. 2005). ANR is a DFR-like reductase using $\mathrm{NADH} / \mathrm{NADPH}$ as coenzyme to convert anthocyanidins to two isomers of flavan-3-ols, e.g. (-)-epicatechin and (-)-catechin (Devic et al. 1999; Xie et al. 2003). In vitro experiments showed that ANR bound NADPH and NADH with $\mathrm{Km}$ values of $0.5 \mathrm{mM}$ and $1.0 \mathrm{mM}$ respectively (Xie et al. 2004b). In addition, our previous studies demonstrated the constitutive expression of the enzyme and its function to using in vivo NADPH/NADH as co-factor (Xie et al. 2006). The enzyme was recently localized to the cytoplasm as predicted (Pang et al. 2007). The concentration of cytosolic NADPH/NADH in tobacco plants is unknown, but a report showed that the cellular NADPH concentrations during photosynthesis were estimated to range from 0.1 to $0.5 \mathrm{mM}$ in the blue-green algae Anacystis nidulans (Grossman and McGowan 1975 ). We hypothesize that the presence of ANR in transgenic tobacco plants may alter metabolic homeostasis of NAD or NADP metabolism as the observed DFR expression in rice (Hayashi et al. 2005). Thus, metabolism may be impacted by the changes of cellular homeostasis of NADPH/NADH. For example, sugar alcohol biosynthesis in celery is thought to be regulated by cytosolic NADPH levels (Gao and Loescher 2000). Consequently, the alterations of tissue-dependent patterns of numerous compounds observed in our experiment, e.g. trichomes-secreted cembranoids, likely result from the changes of coenzyme homeostasis or other factors.

\subsection{Impacts of the coupled expression of PAP1 and ANR transgenes on metabolic profiles}

The twenty-four metabolite profiles altered in the PAP1::ANR transgenic F1 progeny plants were also impacted in ANR or PAP1 transgenic plants (Figs. 5 and 6). It was interesting that obviously overlaid properties of metabolic profiles were observed between PAP1 or ANR and PAP1::ANR plants (Fig. 5, Fig. 6). Without considering the effects of tissue specificity, it was observed that the accumulation levels of eleven identical metabolites, i.e. pentacosane, ascorbic acid, citramalic acid, glucuronic acid, oxalic acid, pyroglutamic acid, tartaric acid, trehalose, trihydroxybutyric acid, and xylitol, which were altered in PAP1::ANR transgenic plants, were increased or decreased in PAP1 or/and ANR transgenic plants (Figs. 5 and 6). Twenty three of the twenty-four metabolites, which were showed their accumulation pattern alterations in PAP1::ANR transgenic plants, were also with altered levels in PAP1 transgenic plants (Figs. 5 and 6).

It was interesting that a counteracting consequence on metabolite accumulation patterns was observed in PAP1::ANR transgenic plants due to the co-expression of PAP1 and ANR. On the one hand, metabolite accumulation patterns altered in ANR transgenic plants were counteracted by the expression of PAP1 in PAP1::ANR transgenic plants. Eleven metabolites with altered levels in $A N R$ transgenic plants were not found to have the alteration in PAP1::ANR transgenic plants. For example, the levels of cembratriene-diol III, IV, VI and VII were increased in ANR transgenic stems, but in PAP1::ANR transgenic stems, reduced to 
similar levels as the ones in WT plants (Fig. 5). In addition, the levels of cembratriene-ol I, cholesterol, citramalic acid, and xylitol were increased in old leaves of the ANR transgenic plants but were reduced in the PAP1::ANR transgenic plants (Fig. 5). Other examples include citric acid, oxalic acid, ascorbic acid, and citramalic acid (Fig. 5). On the other hand, metabolite accumulation alterations caused by the expression of $P A P 1$ were counteracted by the expression of ANR. Levels of thirty-nine metabolites were altered in PAP1 transgenic plants, among which the alteration of the levels of fifteen metabolites was diminished in the PAP1::ANR transgenic plants (Fig.5). For example, the levels of glucose-6-phosphate and glucose were reduced in PAP1 transgenic stems, but in PAP1 $\times$ ANR transgenic stems, returned to the normal levels as the ones in WT plants (Fig. 5). No evidence has been reported describing counteracting impacts of the co-expression of a regulatory gene and a structure gene from the flavonoid pathway on cellular metabolomes. We suggest that this phenomenon results from the reduction of transgene dosage in PAP1::ANR F1 progeny plants. Given that PAP1 transgenic plants were homozygotic plants, the transgene dosage was higher in the mother plants than PAP1::ANR F1 progeny plants. In addition, the mechanism of counteracting phenomenon is likely more complicated than the additive of the two transgenes. This may be also due to the metabolon formation (metabolic channeling) consisting of multiple proteins required for every single secondary metabolite and for avoiding metabolic interference (Jorgensen et al. 2005; Winkel-Shirley 1999).

\section{Conclusion}

The PAP1 gene encodes a R2R3-MYB transcription factor and its ectopic expression led to the formation of a complete anthocyanin pathway in all tissues of tobacco (Nicotiana tobacum) plants. Anthocyanidin reductase (ANR) catalyzes anthocyanidins to flavan-3-ols and its ectopic expression of $A N R$ formed an incomplete pathway of flavan-3-ols and proanthocyanidins (PAs) in leaves and stems of tobacco plants. The co-expression of PAP1 and ANR constructed a complete biosynthetic pathway of PAs in tobacco leaves, stems and flowers. Gas chromatography-mass spectrometry (GC-MS) analysis identified eighty-seven metabolites in wild-type tobacco leaves and stems, and revealed tissue-specific and development-dependent patterns of the abundance of seventy-one metabolites in plants. In PAP1 transgenic plants, the accumulation patterns of thirty-nine metabolites (out of eightyseven) including both primary and secondary metabolites were altered in either leaves or stems. In PAP1::ANR transgenic plants, twenty-four metabolites showed accumulation pattern alterations. Shikimic acid, phenylalanine, and malonic acid, which are three early precursors of the flavonoid pathway, were altered in their abundance in these two transgenic events. In ANR transgenic plants, nineteen metabolites showed abundance alterations. The array of metabolites altered in ANR transgenic plants was distinct from that in PAP1 transgenic plants. The array of metabolites altered in PAP1::ANR transgenic plants were intermediate to the two transgenic events. Principal component analysis showed that there was close ordination relevance in metabolic profile alterations between PAP1 alone and PAP1::ANR transgenic plants but distant ordination relevance between PAP1 and ANR transgenic plants or wild-type plants. These results indicated that the ectopic expression of $P A P 1, A N R$, or PAP1::ANR leading to different branch pathways of plant flavonoids differentially impacted accumulation patterns of metabolites. 


\section{Acknowledgments}

The completion of this work benefited from the support of the Noble Foundation and the support of an USDA grant (USDA 2006-35318-17431). We appreciate constructive advice provided by Lloyd Sumner and Richard Dixon. We appreciate Dr. Bettina E. Broeckling for her critical reading and suggestions.

\section{References}

Borevitz JO, Xia Y, Blount J, Dixon RA, Lamb C (2000) Activation tagging identifies a conserved MYB regulator of phenylpropanoid biosynthesis. Plant Cell 12: 2383-2394

Broeckling CD, Huhman DV, Farag MA, Smith JT, May GD, Mendes P, Dixon RA, Sumner LW (2005) Metabolic profiling of Medicago truncatula cell cultures reveals the effects of biotic and abiotic elicitors on metabolism. J. Exp. Bot. 56: 323-336

Broeckling CD, Reddy IR, Duran AL, Zhao X, Sumner LW (2006) MET-IDEA: Data extraction tool for mass spectrometry-based metabolomics. Anal. Chem. 78: 4334-4341

Choi YE, Harada E, Wada M, Tsuboi H, Morita Y, Kusano T, Sano H (2001) Detoxification of cadmium in tobacco plants: formation and active excretion of crystals containing cadmium and calcium through trichomes. Planta 213: 45-50

Devic M, Guilleminot J, Debeaujon I, Bechtold N, Bensaude E, Koornneef M, Pelletier G, Delseny M (1999) The BANYULS gene encodes a DFR-like protein and is a marker of early seed coat development. Plant J 19: 387-398

Gao Z, Loescher WH (2000) NADPH supply and mannitol biosynthesis. Characterization, cloning, and regulation of the non-reversible glyceraldehyde-3-phosphate dehydrogenase in celery leaves. Plant Physiol. 124: 321-330

Gonzalez A, Mendenhall J, Huo Y, Lloyd A (2009) TTG1 complex MYBs, MYB5 and TT2, control outer seed coat differentiation. Develop Bio 325: 412-421

Grossman A, McGowan RE (1975 ) Regulation of glucose 6-phosphate dehydrogenase in bluegreen algae Plant Physiol. 55: 658-662

Halket J, Przyborowska A, Stein S, Mallard W, Down S, Chalmers R, .. . . (1999) Deconvolution gas chromatography/mass spectrometry of urinary organic acids--potential for pattern recognition and automated identification of metabolic disorders. Rapid Commun Mass Spectrom 13: 279-284

Hayashi M, Takahashi H, Tamura K, Huang J, Yu L-H, Kawai-Yamada M, Tezuka T, Uchimiya H (2005) Enhanced dihydroflavonol-4-reductase activity and NAD homeostasis leading to cell death tolerance in transgenic rice. PNAS 102: 7020-7025

Jorgensen K, Rasmussen AV, Morant M, Nielsen AH, Bjarnholt N, Zagrobelny M, Bak S, Moller BL (2005) Metabolon formation and metabolic channeling in the biosynthesis of plant natural products. Cur. Opin.Plant Biol. 8: 280-291

Korth KL, Doege SJ, Park S-H, Goggin FL, Wang Q, Gomez SK, Liu G, Jia L, Nakata PA (2006) Medicago truncatula mutants demonstrate the role of plant calcium oxalate crystals as an effective defense against chewing insects. Plant Physiol. 141: 188-195

Lillo C, Lea US, Ruoff P (2008) Nutrient depletion as a key factor for manipulating gene expression and product formation in different branches of the flavonoid pathway. Plant Cell Environ 31: 587-601

Ouakfaoui SE, Miki B (2005) The stability of the Arabidopsis transcriptome in transgenic plants expressing the marker genes nptII and uidA. Plant J. 41: 791-800

Pang Y, Peel GJ, Wright E, Wang Z, Dixon RA (2007) Early steps in proanthocyanidin biosynthesis in the model legume Medicago truncatula. Plant Physiol 145: 601-615 
Ramsay NA, Glover BJ (2005) MYB-bHLH-WD40 protein complex and the evolution of cellular diversity. Trends Plant Sci 10: 63-70

Rowan DD, Cao M, Kui L-W, Cooney JM, Jensen DJ, Austin PT, Hunt MB, Norling C, Hellens RP, Schaffer RJ, Allan AC (2009) Environmental regulation of leaf colour in red 35S:PAP1 Arabidopsis thaliana. New Phytologist 182: 102-115

Sarret G, Harada E, Choi Y-E, Isaure M-P, Geoffroy N, Fakra S, Marcus MA, Birschwilks M, Clemens S, Manceau A (2006) Trichomes of tobacco excrete zinc as zinc-substituted calcium carbonate and other zinc-containing compounds. Plant Physiol. 141: 1021-1034

Saxena D, Stotzky G (2001) Bt corn has a higher lignin content than non-Bt corn. Am. J. Bot. 88: 1704-1706

Shi M-Z, Xie D-Y (2010) Features of anthocyanin biosynthesis in pap1-D and wild-type Arabidopsis thaliana plants grown in different light intensity and culture media conditions. Planta 231: 1385-1400

Shi M-Z, Xie D-Y (2011) Engineering of red cells of Arabidopsis thaliana and comparative genome-wide gene expression analysis of red cells versus wild-type cells Planta DOI: 10.1007/s00425-010-1335-2: In press

Springob K, Nakajima H, Yamazaki M, Saito K (2003) Recent advances in the biosynthesis and accumulation of anthocyanins. Nat. Prod. Rep. 20: 288-303

Tanner GJ, Francki KT, Abrahams S, Watson JM, Larkin PJ, Ashton AR (2003) Proanthocyanidin biosynthesis in plants. Purification of legume leucoanthocyanidin reductase and molecular cloning of its cDNA. J Biol Chem 278: 31647-31656

Tohge T, Nishiyama Y, Hirai MY, Yano M, Nakajima J, Awazuhara M, Inoue E, Takahashi H, Goodenowe DB, Kitayama M, Noji M, Yamazaki M, Saito K (2005) Functional genomics by integrated analysis of metabolome and transcriptome of Arabidopsis plants over-expressing an MYB transcription factor. Plant J 42: 218-235.

VolkA GM, Franceschi VR (2000) Localization of a calcium channel-like protein in the sieve element plasma membrane. Aust. J. Plant Physiol. 27: 779-786

Wang E, Hall JT, Wagner GJ (2004) Transgenic Nicotiana tabacum L. with enhanced trichome exudate cembratrieneols has reduced aphid infestation in the field. Mol. Breeding 13: 49-57

Wang E, Wang R, DeParasis J, Loughrin JH, Gan S, Wagner GJ (2001) Suppression of a P450 hydroxylase gene in plant trichome glands enhances natural-product-based aphid resistance. Nat Biotech 19: 371-374

Winkel-Shirley B (1999) Evidence for enzyme complexes in the phenylpropanoid and flavonoid pathways. Physiol. Plant. 107: 142-149

Xie D-Y, Jackson LA, Cooper JD, Ferreira D, Paiva NL (2004a) Molecular and biochemical analysis of two cDNA clones encoding dihydroflavonol-4-reductase from Medicago truncatula. Plant Physiol. 134: 979-994

Xie D-Y, Sharma SB, Dixon RA (2004b) Anthocyanidin reductases from Medicago truncatula and Arabidopsis thaliana. Arch Biochem Biophys 422: 91-102

Xie D-Y, Sharma SB, Paiva NL, Ferreira D, Dixon RA (2003) Role of anthocyanidin reductase, encoded by BANYULS in plant flavonoid biosynthesis. Science 299: 396-399

Xie D-Y, Sharma SB, Wright E, Wang Z-Y, Dixon RA (2006) Metabolic engineering of proanthocyanidins through co-expression of anthocyanidin reductase and the PAP1 MYB transcription factor. Plant J 45: 895-907

Zhou L-L, Zeng H-N, Shi M-Z, Xie D-Y (2008) Development of tobacco callus cultures over expressing Arabidopsis PAP1/MYB75 transcription factor and characterization of anthocyanin biosynthesis Planta 229: 37-51 




\author{
Transgenic Plants - Advances and Limitations \\ Edited by PhD. Yelda Ozden Çiftçi
}

ISBN 978-953-51-0181-9

Hard cover, 478 pages

Publisher InTech

Published online 07, March, 2012

Published in print edition March, 2012

Development of efficient transformation protocols is becoming a complementary strategy to conventional breeding techniques for the improvement of crops. Thus, Transgenic Plants - Advances and Limitations covers the recent advances carried on improvement of transformation methods together with assessment of the impact of genetically transformed crops on biosafety. Each chapter has been written by one or more experienced researchers in the field and then carefully edited to ensure throughness and consistency.

\title{
How to reference
}

In order to correctly reference this scholarly work, feel free to copy and paste the following:

Corey D. Broeckling, Ke-Gang Li and De-Yu Xie (2012). Comparative Metabolomics of Transgenic Tobacco Plants (Nicotiana tabacum var. Xanthi) Reveals Differential Effects of Engineered Complete and Incomplete Flavonoid Pathways on the Metabolome, Transgenic Plants - Advances and Limitations, PhD. Yelda Ozden Çiftçi (Ed.), ISBN: 978-953-51-0181-9, InTech, Available from: http://www.intechopen.com/books/transgenicplants-advances-and-limitations/comparative-metabolomics-of-transgenic-tobacco-plants-nicotiana-tabacumvar-xanthi-reveals-different

\section{INTECH}

open science | open minds

\section{InTech Europe}

University Campus STeP Ri Slavka Krautzeka 83/A 51000 Rijeka, Croatia Phone: +385 (51) 770447 Fax: +385 (51) 686166 www.intechopen.com

\section{InTech China}

Unit 405, Office Block, Hotel Equatorial Shanghai No.65, Yan An Road (West), Shanghai, 200040, China 中国上海市延安西路65号上海国际贵都大饭店办公楼405单元 Phone: +86-21-62489820

Fax: +86-21-62489821 
(C) 2012 The Author(s). Licensee IntechOpen. This is an open access article distributed under the terms of the Creative Commons Attribution 3.0 License, which permits unrestricted use, distribution, and reproduction in any medium, provided the original work is properly cited. 